\title{
Phase Transitions in Nonequilibrium Systems
}

\author{
David Mukamel \\ Department of Physics of Complex Systems, The Weizmann Institute of Science, Rehovot 76100, \\ Israel
}

\section{Introduction}

Collective phenomena in systems far from thermal equilibrium have been a subject of extensive studies in recent years. Usually these systems are driven out of equilibrium by external fields, such as electric field in the case of conductors, pressure gradient in the case of fluids, temperature gradient in the case of heat conductors, chemical potential gradient in the case of growth problems and many others [1] [4]. These driving fields are very common in nature and are found in a large variety of physical systems such as granular and traffic flow

[5] [7], gel electrophoresis [8,9], superionic conductors [10,11] to give a few examples. In many cases these systems reach a steady state, which unlike the equilibrium case, is characterised by non-vanishing currents. In these lectures we consider possible collective phenomena and phase transitions which may take place in such steady states. The main problem in studying nonequilibrium systems is the lack of general theoretical framework within which they could be analysed. As a result they are far less understood as compared with equilibrium systems where the Gibbs picture provides such a theoretical framework.

Before discussing nonequilibrium systems it is useful to consider briefly systems in thermal equilibrium. Here decades of studies have yielded a fairly detailed understanding of their thermodynamic behaviour. Many rules which govern phase transitions occurring in these systems have been derived. For example, it has been shown that the critical exponents associated with a phase transition may be classified into universality classes. These classes do not depend on the detailed interactions in the system but rather on a few parameters 
such as the symmetry of the system and of the order parameter associated with the transition, the dimensionality of the system and the range of interactions. Therefore, in order to study theoretically the critical behaviour of a given system it is sufficient to analyse the simplest possible model which belongs to the same universality class. For reviews see, for example, [12,13]. It has also been shown that phase transitions and spontaneous symmetry breaking do not take place at low dimension. In particular, no phase transition is expected to take place in a one dimensional system at finite temperatures as long as the interactions are short range [14]. Moreover, breaking of continuous symmetry may take place under the same conditions only in dimensions higher than two [15]. Other rules derived by Landau relate the nature of the transition, namely whether it is first order or continuous, to the symmetry of the systems [14]. If the symmetry allows a third order term in the expansion of the free energy in the order parameter, such as in the case of the transition from a liquid to a nematic liquid crystal phase [16], the transition cannot be continuous and is necessarily first order. On the other hand if the symmetry is such that no third order term is allowed, such as in the transition from a paramagnetic to a ferromagnetic phase, the transition may either be first order or continuous, depending on the details of the interactions. The Gibbs phase rule is another very useful example of a rule which governs the phase digrams of systems in equilibrium [14]. It deals with fluids composed of $c$ components. The thermodynamic phase space of such systems is of $c+1$ dimensions, associated with the temperature, pressure, and $c-1$ chemical potentials. According to the rule, the manifold in this space on which $n$ different phases coexist is of $D=2+c-n$ dimensions. Another rule deals with the phase diagram near a triple point, where three coexistence lined meet. According to this $180^{\circ}$ rule, each of the three angles defined by the intersecting coexistence lines must be less than $180^{\circ}$. This is a direct result of the convexity of the free energy [17].

These rules and many others, some of which are related to disordered systems [18,19, provide extremely useful tools for analysing and understanding phase diagrams and critical behaviour of models and physical systems in equilibrium. By simply identifying the symmetry of the system and the nature of the order paramenter involved in the phase transition 
one can usually find the universality class of the transition and even obtain a rough idea of the possible phase diagram.

Our degree of understanding of collective behaviour far from thermal equilibrium is at a much more primitive stage. Since a general theoretical framework for studying nonequilibrium phenomena does not exist, one cannot derive similar rules which would be as general as those for equilibrium systems. Rather, one has to resort to studying specific models and probe the resulting types of phase diagrams and phase transitions, with the hope that some general picture might emerge.

In the present lectures we consider stochastic driven systems in one dimension and discuss some interesting collective behaviour which they display. Unlike equilibrium one dimensional systems which do not exhibit phase transitions, non equilibrium systems exhibit a rich variety of collective phenomena such as first order and continuous phase transitions, spontaneous symmetry breaking (SSB), phase separation, slow coarsening processes and many others. Mechanisms which lead to these phenomena are discussed.

The article is organised as follows: in Section III the concept of detailed balance is discussed, the lack of which is characteristic of nonequilibrium systems. A necessary and sufficient condition for the existence of detailed balance is presented. In Section ПII a simple driven model, the totally asymmetric exclusion process, is introduced and its phase diagram for a system with open boundaries is calculated using a mean field approximation. The phase diagram exhibits several phases separated by first order and continuous transitions. The matrix method which enables one to obtain exact results for steady state properties is outlined in Section IV. A model which displays spontaneous symmetry breaking in one dimension is introduced in Section $\nabla$ and a model exhibiting phase separation accompanied by slow coarsening processes is described in Section V1. Open problems and perspectives are briefly discussed in Section VII. 


\section{Detailed balance and driven systems}

In this section we make some general considerations concerning the evolution of dynamical systems. Let $C$ be a microscopic configuration, and let $P(C, t)$ be the probability that the system is in the microscopic configuration $C$ at time $t$. The dynamics of the system is defined in terms of the transition rates $W\left(C \rightarrow C^{\prime}\right)$ from a configuration $C$ to $C^{\prime}$. The equation which governs the evolution of the distribution function $P(C, t)$ takes the form

$$
\frac{\partial P(C, t)}{\partial t}=\sum_{C^{\prime}} W\left(C^{\prime} \rightarrow C\right) P\left(C^{\prime}, t\right)-\sum_{C^{\prime}} W\left(C \rightarrow C^{\prime}\right) P(C, t) .
$$

The first sum represents the rate of flow, in configuration space, of probability into $C$ while the second sum corresponds to the outgoing flow from this configuration. In a steady state the two terms are equal, yielding zero net flow from any configuration.

Systems in thermal equilibrium are characterised by an energy function, or a Hamiltonian, $E(C)$. The steady state distribution $P(C)$ is proportional to $e^{-E(C) / k_{B} T}$, where $T$ is the temperature and $k_{B}$ is the Boltzmann constant. Given an energy function $E(C)$ one can always find transition rates $W\left(C \rightarrow C^{\prime}\right)$, such as the Metropolis rates, which obey detailed balance. Here the two sums cancel term by term

$$
W\left(C^{\prime} \rightarrow C\right) P\left(C^{\prime}\right)=W\left(C \rightarrow C^{\prime}\right) P(C)
$$

for any pair of configurations $C$ and $C^{\prime}$.

On the other hand dynamical systems are not defined by an energy function but rather by transition rates. When a system is not in thermal equilibrium, the resulting steady is such that detailed balance (2) is not satisfied. We will basically use this lack of detailed balance as a definition of nonequilibrium.

Given the dynamics of a system, namely the transition rates, it is of interest to know whether or not detailed balance is satisfied. Since, in general, the steady state distribution cannot be calculated, a direct check of the detailed balance condition (2) is not possible. Thus a criterion for existence of detailed balance which is based directly on the transition 
rates and does not require the knowledge of the steady state is highly desirable. Such a criterion is provided by the following equations. Let $C_{1}, C_{2}, \ldots, C_{k}$ be a set of $k$ microscopic configurations. A necessary and sufficient condition for the existence of detailed balance is that for any such set one has

$$
W(1 \rightarrow 2) W(2 \rightarrow 3) \ldots W(k \rightarrow 1)=W(1 \rightarrow k) W(k \rightarrow k-1) \ldots W(2 \rightarrow 1)
$$

where for simplicity we have denoted $C_{i}$ by $i$. It is easy to check that (3) is a necessary condition. When detailed balance is satisfied one may replace $W(i \rightarrow i+1) / W(i+1 \rightarrow i)$ by $P(i+1) / P(i)$, where $\mathrm{P}(\mathrm{i})$ is the steady state distribution with respect to which detailed balance is satisfied. Using these relations (3) is easily verified.

To demonstrate that this is a sufficient condition as well, we use (3) to derive the steady state distribution. We start with an arbitrary configuration 1 and denote its steady state weight by $P(1)$. The weight of states 2 which are directly connected with 1 (namely, for which $W(1 \rightarrow 2)>0)$, may thus be defined using the detailed balance relation, $P(2)=$ $P(1) W(1 \rightarrow 2) / W(2 \rightarrow 1)$. This process may then be repeated to define the weights of states directly connected with states 2 etc, until all microscopic configurations have been reached. The weight of a microscopic configuration $k$ which may be reached from 1 via intermediate states $2,3, \ldots, k-1$ is thus given by

$$
P(k)=P(1) \frac{W(1 \rightarrow 2) \ldots W(k-1 \rightarrow k)}{W(k \rightarrow k-1) \ldots W(2 \rightarrow 1)} .
$$

For this procedure to be self-consistent one has to verify that any path between configurations 1 and $k$ yields the same $P(k)$. It is a straightforward matter to show that this follows directly from (3).

Thus, to demonstrate that a dynamical system defined by its transition rates is not in thermal equilibrium it is sufficient to find a single path in configuration space for which (3) is not satisfied. This is usually quite easy to check, making it a very useful criterion. When (3) is not satisfied the system exhibits non-vanishing probability currents between configurations which is an indication of the system not being in thermal equilibrium. 
A simple prototypical model of driven systems, termed the 'standard model', was introduced by Katz et al [20,1]. This is a driven lattice gas model defined on a hypercubic lattice with periodic boundary conditions. Each site $i$ is either occupied by a particle or is vacant, with $\sigma_{i}=0,1$ being the occupation number. In the absence of drive, an Ising Hamiltonian is assumed

$$
H=-J \sum_{\langle i j\rangle} \sigma_{i} \sigma_{j}
$$

where the sum is over nearest neighbour (nn) sites $\langle i j\rangle$. The evolution of the system is defined by Kawasaki dynamics, allowing for particles to hop between nearest neighbour sites. Let $C$ and $C^{\prime}$ be two configurations obtained from each other by an interchange of a single pair of nn occupation numbers $\sigma_{i}$ and $\sigma_{j}$. The transition rate between $C$ and $C^{\prime}$ may be taken as the Metropolis rate $W\left(C \rightarrow C^{\prime}\right)=w(\beta \Delta H)$, where $\Delta H=H\left(C^{\prime}\right)-H(C)$ and $w(x)=$ $\min \left(1, e^{-x}\right)$. This dynamics leads to the expected Boltzmann equilibrium distribution. In $d>1$ dimensions the system exhibits the usual Ising transition from a homogeneous phase at high temperatures to a phase separated state at low temperatures.

Introducing a driving field $E$ along one of the axes, the transition rates are modified by adding a term $u E$ to $\Delta H$ where

$$
u=-1,0,+1
$$

for a hop along, transverse or opposite to the field direction, respectively. The transition rates are thus given by

$$
W\left(C \rightarrow C^{\prime}\right)=w(\beta(\Delta H+u E)) .
$$

Due to the periodic boundary conditions in the direction of the driving field, these rates do not obey detailed balance, and the steady exhibits non-vanishing currents.

In spite of the simplicity of the model, no exact results for the steady state properties are available (exact in one dimension). Extensive numerical studies of this model in two dimensions demonstrate that the phase separation transition which exists in zero drive, 
persists for non-zero driving fields. At low temperatures the system exhibits stripes of high density and low density regions which are oriented along the field direction. These stripes coarsen with time leading to a phase separated state.

\section{Asymmetric exclusion process in one dimension}

In this section we consider the phase diagram of the one dimensional 'standard model' defined in the previous section for $J=0$. Here the only interaction between the particles is the hard-core interaction which prevents more than one particle from occupying the same site. This process is called asymmetric simple exclusion process (ASEP). Furthermore, we consider the limit $E \rightarrow \infty$, called totally asymmetric simple exclusion process (TASEP). In this limit particles are restricted to move only to the right, with no backward moves. This model turns out to be sufficiently simple to allow for exact calculation of some of its steady state properties. In spite of its simplicity, the model with open boundary conditions exhibits a rather rich and complicated phase diagram, displaying both continuous and discontinuous phase transitions (see below). This is clearly a direct consequence of the fact that the dynamics is a nonequilibrium one. The model and many variants of it have been a subject of extensive studies in recent years [21 30].

The dynamics of the model is defined as follows: at any given time a pair of nn sites is chosen at random. If the occupation numbers of these sites are $(+0)$ an exchange is carried out

$$
+0 \rightarrow 0+
$$

with rate 1 . All other configurations, remain unchanged. Here and in the following we interchangably use 1 or + to denote an occupied site. For periodic boundary conditions the system reaches a trivial steady state in which all microscopic configurations have the same weight. This may be verified by direct inspection of the master equation (11). It is

easy to see that the number of configurations to which a given configuration $C$ may flow 
is equal to the number of configurations flowing into $C$. To verify that this is the case note that a microscopic configuration $C$ is composed of alternating segments of $+^{\prime} s$ and $0^{\prime} s$. According to the dynamics (8) $C$ may be exited when the rightmost particle in one of the + segments moves one step to the right. Thus the number of configurations $C$ may flow into is equal to $l$, the number of + segments in this configuration. Similarly $C$ may be reached when the leftmost particle in one of the + segments hops into its position. The number of configurationd flowing into $C$ is therefore also equal to $l$. Since all non-vanishing transition rates are 1, the state where all configurations have equal weights is stationary. Therefore in the steady state the system exhibits no correlations, apart from the trivial correlations arising from the fact that the overall density of particles is fixed.

The steady state current $J$ is given by

$$
J=\left\langle\sigma_{i}\left(1-\sigma_{i+1}\right)\right\rangle,
$$

where the brackets denote a statistical average with respect to the steady state weights of the microscopic configuration. Since in the steady state the system exhibits no correlations the current may be written, in the large system limit, as

$$
J=p(1-p)
$$

where $p_{i}=\left\langle\sigma_{i}\right\rangle$ is the density at site $i$, and the index $i$ is omitted in (10) since the average density is homogeneous, independent of $i$. Equation (10), relating the current to the density is known as the fundamental relation (or fundamental diagram). The interesting feature in this relation is that the current is not a monotonic function of the density but rather it exhibits a maximum at $p=0.5$. This feature is a result of the hard-core interaction between the particles and it affects rather drastically the steady state properties of the system when open boundary conditions are considered.

We now turn to the model with open boundary conditions. Here, particles are introduced into the system at the left end, they move through the bulk according to the conserving dynamics (8), and leave the system at the right end. To be more specific, at the left boundary $(i=1)$ the move 


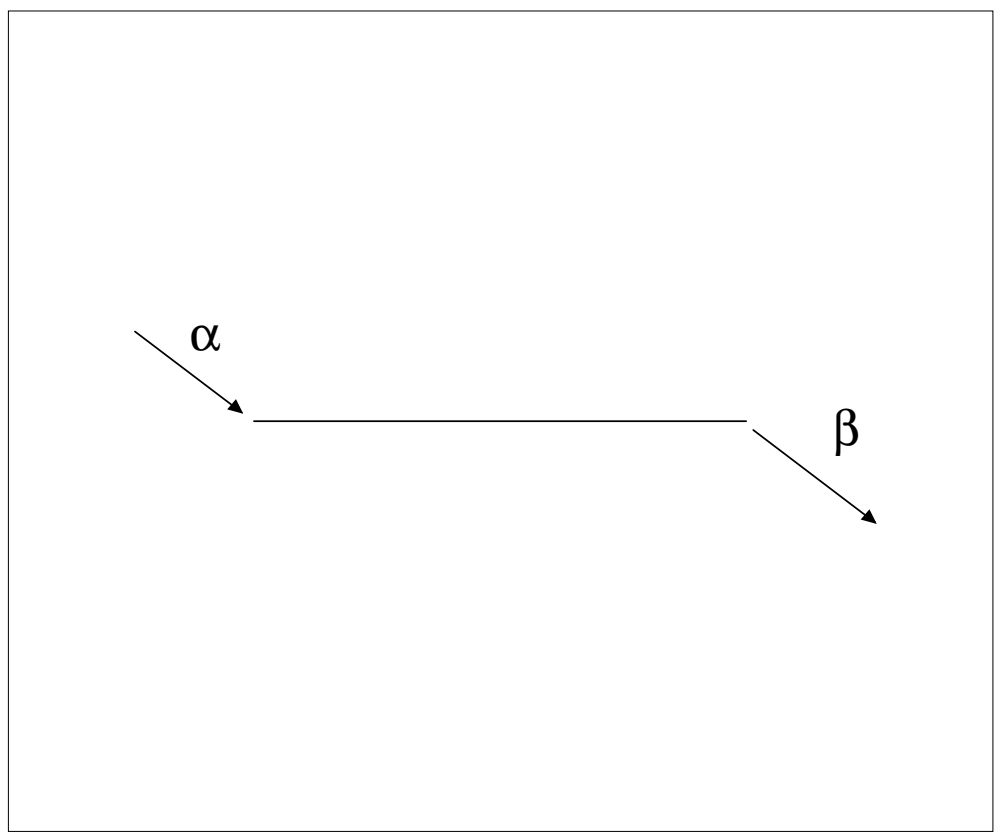

FIG. 1. A schematic representation of the totally asymmetric exclusion process with open boundary conditions.

$$
0 \rightarrow+
$$

is carried out with a rate $\alpha$. Similarly, at the right boundary $(i=N)$ one takes

$$
+\rightarrow 0
$$

with a rate $\beta$. For a schematic representation of the model see Figure 11. The overall dynamics is non-conserving. Particles are conserved in the bulk but are not conserved at the boundaries. Unlike the case with periodic boundary conditions, the steady state distribution is not trivial, and correlations between the densities at different sites do not vanish. However, far from the boundaries the distribution function is expected to be well approximated by the homogeneous one, suggesting that correlations are small.

We are interested in the steady state of this model for given rates $\alpha$ and $\beta$. For large $\alpha$ and small $\beta$, namely for a large feeding rate and a small exit rate the overall density is expected to be high. On the other hand for small $\alpha$ and large $\beta$ the density is expected to be low. In addition, for a large system, where away from the boundaries the local density is expected to vary very slowly, the fundamental relation (10) is expected to hold locally. Thus 
the current in the system cannot exceed a maximal current, as suggested by (10). These features yield a rather rich phase diagram, as $\alpha$ and $\beta$ are varied.

We start by considering the phase diagram in the mean field approximation [21,22, 24]. Since correlations in this system are expected to be vanishingly small away from the boundaries, this approximation is expected to yield a rather accurate phase diagram. In fact it turns out that the phase diagram obtained in this way is exact.

To derive the mean field equations we note that the current $J_{i, i+1}$ between sites $i$ and $i+1$ is given by $\left\langle\sigma_{i}\left(1-\sigma_{i+1}\right)\right\rangle$ for $i=1, \ldots, N-1$. In addition the currents at the two ends are given by $J_{0}=\alpha\left\langle 1-\sigma_{1}\right\rangle$ and $J_{N}=\beta\left\langle\sigma_{N}\right\rangle$. Neglecting correlations one finds that in the steady state, where all currents are equal, the following equations have to be satisfied:

$$
J=\alpha\left(1-p_{1}\right)=p_{1}\left(1-p_{2}\right)=\ldots=p_{N-1}\left(1-p_{N}\right)=\beta p_{N} .
$$

Solving these equations for $J, p_{1}, \ldots, p_{N}$ the density profile in the steady state and the current are obtained.

It is instructive to consider these equations in the continuum limit. Replacing $p_{i}$ by $p(x)$ in (13) with $0 \leq x \leq L$ yields the bulk current

$$
J(x)=p(1-p)-D \frac{\partial p}{\partial x},
$$

where $D$ is the diffusion constant which, by rescaling $x$, may be taken as 1 . In this expression the first term represents the drive while the second term is the ordinary diffusion current. The evolution of the system is governed by the continuity equation $\partial p / \partial t=-\partial J / \partial x$, together with the boundary conditions $J(0)=\alpha(1-p(0))$ and $J(L)=\beta p(L)$. In the steady state the current $J$ in (14) is independent of $x$ yielding a density profile which has one of the two following forms

$$
\begin{aligned}
& p(x)=0.5+v \tanh \left[v\left(x-x_{0}\right)\right] \\
& p(x)=0.5+v \operatorname{coth}\left[v\left(x-x_{0}\right)\right],
\end{aligned}
$$

where $v^{2}=1 / 4-J$. The two parameters $x_{0}$ and $v$ (or alternatively the current $J$ ) are determined by the two boundary conditions, and are thus related to $\alpha$ and $\beta$. 
By matching the boundary conditions the density profiles and the current are obtained. The resulting phase diagram is given in Figure 2. The system is found to exhibit three distinct phases in the limit of large length $L$ :

- Low density phase for which the bulk density is smaller than 0.5 with $x_{0}=O(L)$. The density profile is basically flat, except for a small region near the right end. In this phase $p(0)=\alpha$ and $J=\alpha(1-\alpha)$. It exists for $\alpha<\beta$ and $\alpha<1 / 2$.

- High density phase with bulk density larger than 0.5 with $x_{0}=-O(L)$. The density profile is flat except at a small region near the left end. Here $p(L)=1-\beta$ and $J=\beta(1-\beta)$. This phase exist for $\beta<\alpha$ and $\beta<1 / 2$. The two phases co-exist on the line $\alpha=\beta<1 / 2$.

- A maximal current phase in the region $\alpha>1 / 2$ and $\beta>1 / 2$. Here the bulk density is $1 / 2$ exhibiting structures at both ends of the system. These structures decay algebraically as $1 / x$ when moving away from the ends. In this phase the current is maximal, namely $J=1 / 4$.

The phase diagram exhibits a first order line on which the high density and the low density phases coexist and two second order lines separating these phases from the maximal current phase. Typical schematic density profiles in the various phases are also given in Figure 2.

In the mean field approximation fluctuations are neglected, and therefore by itself this analysis may not serve as a demonstration that phase transitions do take place in $1 d$ away from thermal equilibrium. In fact mean field approximation yields phase transitions in equilibrium $1 d$ systems, where they are known not to exist. It is therefore important to examine the role of fluctuations in this driven system and demonstrate that indeed the phase transitions found within the mean field approximation remain when fluctuations are taken into account. This has indeed been demonstrated for the TASEP [24 26]. A method 


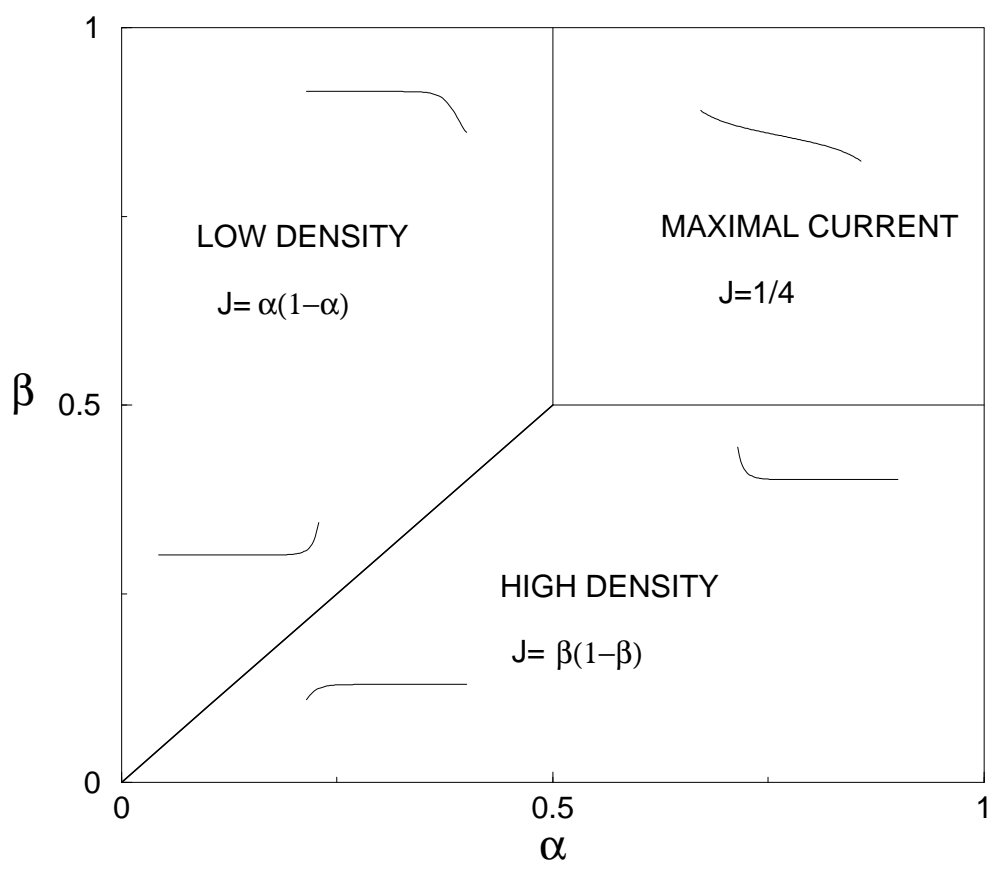

FIG. 2. The $(\alpha, \beta)$ phase diagram of the totally asymmetric exclusion process exhibiting low density, high density and maximal current phases. The thick line represents a first order transition while thin lines correspond to continuous transitions. Schematic density profiles in the various phases are given.

which goes beyond the mean field approximation and allows exact calculations of steady state properties of some driven $1 d$ models is described in the next section.

\section{Matrix method}

A matrix method for calculating some steady state properties of the TASEP was introduced a few years ago [25]. The method has since then been generalised and applied to other models of driven systems. In the following we briefly outline the method as applied to the TASEP with open boundary conditions described above.

We are interested in calculating the steady state distribution function $P\left(\sigma_{1}, \ldots, \sigma_{N}\right)$. In the matrix method one tries to express the distribution function by a matrix element of a product of particular matrices. Let $D$ and $E$ be two square matrices and $\langle W|$ and $|V\rangle$ be two vectors. For any given configuration, $\left(\sigma_{1}, \ldots, \sigma_{N}\right)$ one considers the matrix product in 
which each occupation number $\sigma_{i}$ is replaced by either a matrix $D$ or a matrix $E$ depending on whether it is 1 or 0 , respectively. The key question is whether one can find matrices $D$ and $E$ and vectors $\langle W|$ and $|V\rangle$ such that $P\left(\sigma_{1}, \ldots, \sigma_{N}\right)$ is proportional to the $\langle W|| V$, matrix element of this product. Within this representation the distribution function may be written as

$$
P\left(\sigma_{1}, \ldots, \sigma_{N}\right) \propto\left\langle W\left|\prod_{i=1}^{N}\left[\sigma_{i} D+\left(1-\sigma_{i}\right) E\right]\right| V\right\rangle .
$$

A priori, it is not at all clear that such representation is available. However, if such representation exists, it may yield a straightforward (though sometimes tedious) way for calculating the distribution function. For example, the density at, say, site $i=1$ may be expressed as

$$
p_{1}=\frac{1}{Z_{N}} \sum_{\left\{X_{i}\right\}}\left\langle W\left|D X_{2} \ldots X_{N}\right| V\right\rangle
$$

where $X_{i}=D, E$, and the normalization factor $Z_{N}$ is given by

$$
Z_{N}=\sum_{\left\{X_{i}\right\}}\left\langle W\left|X_{1} X_{2} \ldots X_{N}\right| V\right\rangle=\left\langle W\left|C^{N}\right| V\right\rangle
$$

with

$$
C=D+E
$$

Thus, we may rewrite (17) as

$$
p_{1}=\frac{1}{Z_{N}}\left\langle W\left|D C^{N-1}\right| V\right\rangle
$$

Densities at other sites and density-density correlation functions may similarly be expressed by other matrix elements.

The main question at this point is how to find matrices and vectors such that ([16) holds. To this end we consider the local currents in the system. Using the matrix representation, the current between sites $i$ and $i+1(i=1, \ldots, N-1)$ may be expressed as

$$
J_{i, i+1}=\frac{1}{Z_{N}}\left\langle W\left|C^{i-1} D E C^{N-i-1}\right| V\right\rangle
$$


and the currents at the two ends

$$
\begin{aligned}
J_{0} & =\frac{\alpha}{Z_{N}}\left\langle W\left|E C^{N-1}\right| V\right\rangle \\
J_{N} & =\frac{\beta}{Z_{N}}\left\langle W\left|C^{N-1} D\right| V\right\rangle .
\end{aligned}
$$

In the steady state all currents are equal. Taking matrices $D, E$ and vectors $\langle W|| V$,$\rangle which$ satisfy

$$
\begin{aligned}
& D E=D+E \quad(=C) \\
& \alpha\langle W| E=\langle W| \\
& \beta D|V\rangle=|V\rangle,
\end{aligned}
$$

guarantee that all currents are equal, with

$$
J=\frac{Z_{N-1}}{Z_{N}}
$$

The question is whether these relations (23) are sufficient to guarantee that the resulting distribution (16) is a steady state. For (16) to be a steady state one has to make sure that

$$
\partial P\left(\sigma_{1}, \ldots, \sigma_{N}\right) / \partial t=0
$$

for each of the $2^{N}$ microscopic configurations. The relations (23) only directly guarantee that $N+1$ of these $2^{N}$ equations are satisfied. This may suggest that (23) may not be sufficient to guarantee that the steady state distribution is given by (16). However it can be shown, by direct inspection of Equations (25) that (23) yields the steady state of the system.

The problem is thus reduced to first finding matrices and vectors which satisfy (16), and then calculating some matrix elements to obtain, for example, the current $J$. It is straightforward to show that for $\alpha+\beta \neq 1$ the matrices which satisfy (16) have to be of infinite order. Such matrices have been found and the current and density profiles and other correlation functions have been calculated [25]. The resulting phase diagram coincides with that obtained by the mean field approximation, although the density profiles are different. 
For example, in the algebraic, maximal current, phase the local density decays to the bulk density like $1 / \sqrt{x}$ at large distances from the boundary, unlike the mean field result which yields a $1 / x$ profile.

The matrix method proved to be very powerful in yielding steady state properties of TASEP dynamics. It has been applied and generalised to study partially asymmetric exclusion processes (ASEP) [31 33] and models with more than one type of particles [34 37]. In addition, replacing matrices by tensors proved to be useful in some cases [38, 39]. However the method is restricted to one dimension. It is not standard in the sense that it can not be applied to any dynamical model. Moreover, there is no simple way to tell a priori whether or not it may be applicable for a specific model.

\section{Spontaneous symmetry breaking in one dimension}

In this section we consider a simple dynamical model which exhibits spontaneous symmetry breaking (SSB) in $1 d$.

The model may be pictorially described in the following way: consider a narrow bridge connecting two roads. Cars travelling on the bridge in opposite directions do not block each other, although they may slow the traffic flow in both directions. We assume that the two roads leading to the bridge from both sides are statistically identical. Namely the arrival rates of cars at the two ends of the bridge are the same. This system clearly has a right-left symmetry. Thus if this symmetry is not spontaneously broken one would expect that the long time average of the current of cars travelling to the right would be the same as that of cars travelling to the left. The question is whether the bridge is capable of exhibiting breaking of the right-left symmetry, and spontaneously turning itself into a 'one-way' street, where the current in one direction is larger than the current in the other direction. It turns out that this may indeed take place in the limit of a long bridge, demonstrating that SSB may take place in $1 d$ nonequilibrium systems.

To model the 'bridge' problem we generalise the TASEP discussed in the previous section 
[37,40,41]. We consider a $1 d$ lattice of length $N$. Each lattice point may be occupied by either a $(+)$ particle (positive charge) moving to the right, a $(-)$ particle (negative charge) moving to the left or by a vacancy (0). In addition positive (negative) charges are supplied at the left (right) end and are removed at the right (left) end of the system.

The dynamics of the model is defined as follows: at each time step a pair of nearest neighbour sites is chosen and an exchange process is carried out

$$
+0 \rightarrow 0+, \quad 0-\rightarrow-0, \quad+-\rightarrow-+,
$$

with rates 1,1 and $q$, respectively. Furthermore, at the two ends particles may be introduced or removed. At the left boundary $(i=1)$ the processes

$$
0 \rightarrow+\quad, \quad-\rightarrow 0
$$

take place with rates $\alpha$ and $\beta$, respectively. Similarly, at the right boundary $(i=N)$, one has the processes

$$
0 \rightarrow-\quad, \quad+\rightarrow 0
$$

with rates $\alpha$ and $\beta$, respectively (see Figure [3). In the 'bridge' language the boundary terms may be viewed as traffic lights which control the feeding and exit rates at the two ends.

Since the parameters $\alpha$ and $\beta$ are the same on both ends of the systems the dynamics obviously possesses a right-left symmetry. The question of interest is whether or not this symmetry is preserved in the steady state. Clearly, for small $\alpha$ and large $\beta$ the density of particles in the system is expected to be low, the two types of particles do not block each other, and the steady state is expected to be symmetric. On the other hand for $\beta$ much smaller than $\alpha$, particles are blocked in the system, the density is high and it is possible that symmetry breaking takes place.

We start by considering the mean field approximation. It is straightforward to derive the mean field equations for the steady state. They take the form

$$
\begin{aligned}
& J_{+}=p_{i}\left[1-p_{i+1}-(1-q) m_{i+1}\right] \\
& J_{-}=m_{i+1}\left[1-m_{i}-(1-q) p_{i}\right],
\end{aligned}
$$




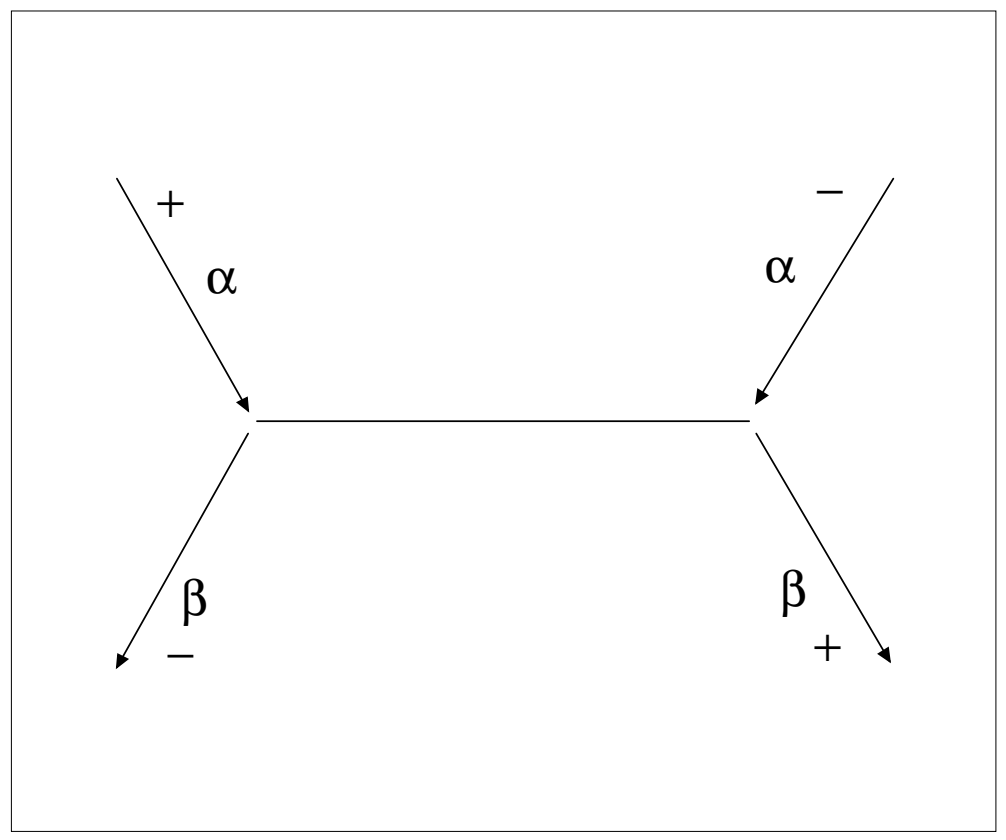

FIG. 3. A schematic representation of the input and output rates of the "bridge' model.

for $i=1, \ldots, N-1$, where $p_{i}$ and $m_{i}$ are the densities of the $(+)$ and $(-)$ particles at site $i$, respectively, and $J_{+}$and $J_{-}$are the currents of the positive and negative particles, respectively. In addition to the bulk equations one has four other equations for the currents at the boundaries

$$
\begin{aligned}
& J_{+}=\alpha\left(1-p_{1}-m_{1}\right)=\beta p_{N} \\
& J_{-}=\beta m_{1}=\alpha\left(1-p_{N}-m_{N}\right) .
\end{aligned}
$$

These $(2 N+2)$ equations may be solved numerically for $\left(p_{1}, \ldots, p_{N} ; m_{1}, \ldots, m_{N} ; J_{+}, J_{-}\right)$to yield the $(\alpha, \beta)$ phase diagram of the model. It is found that for large $\beta$ the steady state is symmetric (with $J_{+}=J_{-}$) while for small $\beta$ the two currents are unequal in the steady state.

The matrix method discussed in the previous section has been generalised and applied to this model [37]. However it turned out that a self-consistent matrix representation could be found for this model only for $\beta=1$ or in the limit $\alpha \rightarrow \infty$. The limit $\alpha \rightarrow \infty$ is trivially mapped on the single species TASEP model. For $\beta=1$ a phase transition is found although no spontaneous symmetry breaking takes place. According to mean field SSB is expected 


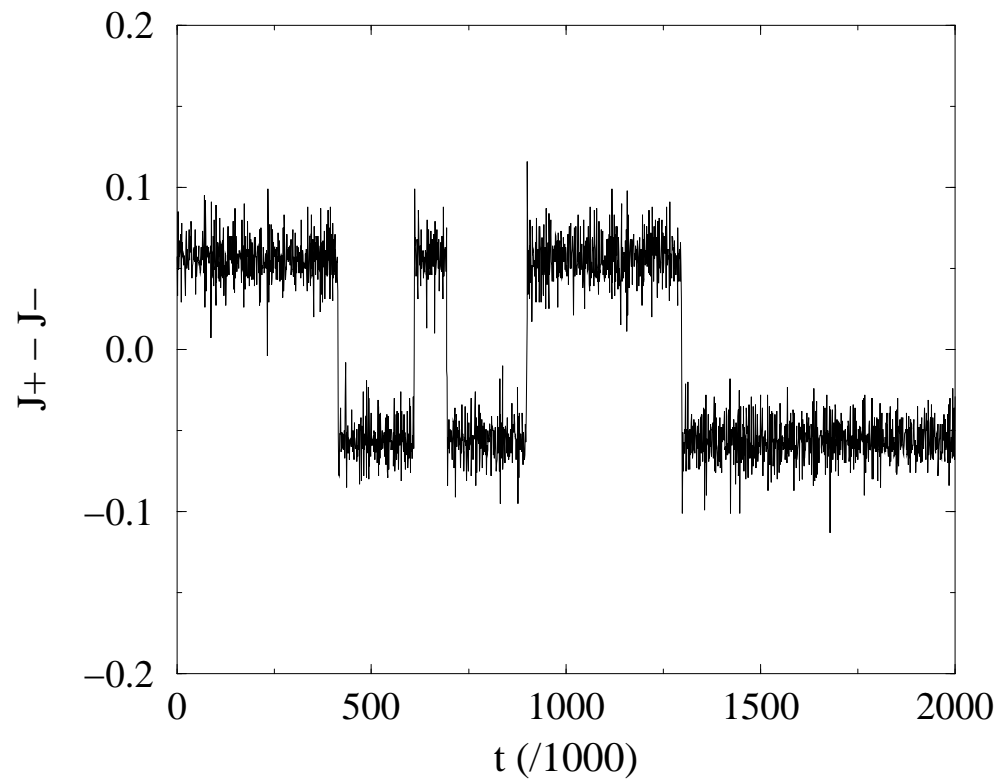

FIG. 4. The time evolution of the current difference in the broken symmetry phase for ( $\alpha=q=1, \beta=0.15, N=80$ ). Time is measured in units of Monte Carlo sweeps. Flips between the two symmetry related states are clealy seen.

only at much lower exit rates $\beta$.

To demonstrate that the non-symmetric state found in the mean field approximation for small $\beta$ survives fluctuations, numerical simulations of the dynamics have been carried out and the current difference $J_{+}-J_{-}$has been measured as a function of time for small $\beta$, where the mean field approximation predicts a broken symmetry phase [37]. A typical time evolution of the current difference for a system of size $N=80$ is given in Figure 4 . The figure suggests that the system flips between two macroscopic states: one with a positive net current and the other with negative net current. In the first case the system is predominantly loaded with positive charges moving to the right while in the second case it is loaded with negative charges moving to the left. This time course is characterised by a time-scale $\tau(N)$ which measures the average time between flips. Clearly, when averaged over time, the current difference vanishes, yielding a symmetric state. This is to be expected since we are dealing with a finite system, and one certainly does not expect SSB to take place in a finite system. The question is how does the system behave in the thermodynamic limit $N \rightarrow \infty$, 


\section{$\frac{----000+\frac{+++++}{\mathrm{x}}}{\mathrm{y}}$}

FIG. 5. A typical microscopic configuration of the "bridge" in the broken symmetry state for small $\beta$. Usually $x$ is a small number of $O(1)$ and $y$ is of $O(N)$ or vise versa.

and particularly how does $\tau(N)$ grow for large $N$. Numerical simulations suggest that $\tau$ grows exponentially with $N$. This means that the probability of a flip is negligibly small in a large system, and thus SSB takes place.

In order to gain some insight into the flipping process we consider the limit of very small $\beta$ 40,42]. In this limit, particles leave the system at a very small rate, and the system is filled with either positive charges moving to the right or negative charges moving to the left. Starting with a positively charged system, one would like to understand the mechanism by which a system of finite length flips into a negatively charged one. The evolution in the small $\beta$ limit may be described as follows: with rate $\beta$ a positive charge leaves the system at the right end. The vacancy created at this end may either move to the left with velocity 1 or may be filled with a negatively charged particle which in turn moves to the left with velocity q. When the negative charge reaches the other end of the system it is delayed for a while, but eventually leaves the system in time of order $1 / \beta$. During this time, other negative charges may arrive at the left end forming a small blockage of negative charges. A typical configuration is given in Figure 5. It is composed of a segment of $x$ negative charges at the left, another segment of $y$ positive charges at the right and in between a segment of $N-x-y$ vacancies. This configuration is denoted by $(x, y)$. In the small $\beta$ limit other configurations, for example those in which vacancies are present inside the charged segments, do not play a role in the global dynamics and may be neglected.

The dynamics restricted to $(x, y)$ configurations is rather simple. It may be viewed as the dynamics of a single particle diffusing on a square lattice performing the following elementary moves:

$$
(x, y) \stackrel{b}{\rightarrow}(x+1, y-1)
$$




$$
\begin{aligned}
& (x, y) \stackrel{a}{\rightarrow}(x, y-1) \\
& (x, y) \stackrel{b}{\rightarrow}(x-1, y+1) \\
& (x, y) \stackrel{a}{\rightarrow}(x-1, y),
\end{aligned}
$$

where

$$
a=\frac{1}{2(1+\alpha)}, \quad b=\frac{\alpha}{2(1+\alpha)}
$$

are the rates of the various moves. Here the first two moves correspond to a positive charge leaving the system at the right end and being replaced by a negative charge (vacancy), respectively. Similarly the last two moves correspond to a negative charge leaving the system at the left end and replaced by a positive charge (vacancy), respectively. A schematic representation of this process is given in Figure 6 .

The biased diffusion process takes place as long as the particle stays within the triangle $(x \geq 0, y \geq 0, x+y \leq N)$. When it reaches the boundary of the triangle, for example $x=0$ the negative charge blockage at the left end disappears and on a very short time scale (as compared with $1 / \beta$ ) the system is filled with positive charges from the left end, moving to $(0, N)$. The evolution of a positively charged system is thus represented by a random walk starting at $(0, N)$ with elementary steps defined by (31). Due to the bias of these elementary steps, a typical walk for large $N$ ends on the $x=0$ axis. Once it reaches this axis it moves back to $(0, N)$ and the process starts again. This process repeats itself until the diffusing particle performs a walk which starts at $(0, N)$ and ends on the $y=0$ axis without touching the $x=0$ axis while diffusing. When this happens the blockage of positive charges at the right end is removed, the system is rapidly filled with negative charges moving to the other end of the triangle $(N, 0)$. This corresponds to a flip. The probability of such a walk taking place has been calculated, yielding the following flipping time [40]:

$$
\tau(N)=\frac{C}{\beta} N^{3 / 2} e^{\kappa N}
$$

where $C$ is a constant and 


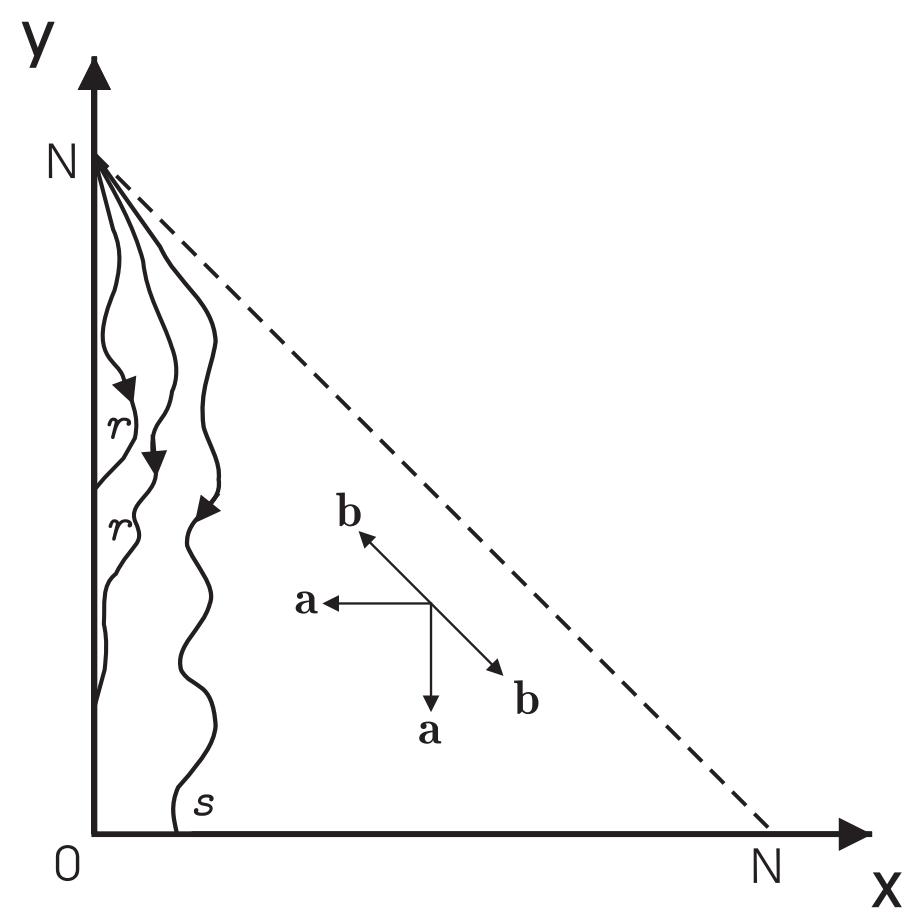

FIG. 6. A representation of the dynamics of the "bridge" in the limit of small $\beta$ as a biased diffusion process on a square lattice. The elementary moves and their rates are indicated. Starting with a positively charged system, namely at $(N, 0)$, possible trajectories are given. Typical walks, $r$, end on the $x=0$ axis. Walks, $s$, which end on the $y=0$ axis are rare, and they correspond to a flip. 


$$
\kappa=\ln \left[\frac{2}{a}(1-a-\sqrt{(1-a)(1-2 a)})\right] .
$$

The exponential flipping time is a direct result of the fact that the random walk corresponding to the evolution of the model is biased. It takes an exponentially long time to reach a distance of order $N$ against a bias.

A very interesting question is related to the behaviour of nonequilibrium systems with spontaneous symmetry breaking when an external symmetry breaking field is introduced. In thermal equilibrium a symmetry breaking field makes the phase unfavoured by the field metastable or even unstable (when the field is large). For example, a positive magnetic field applied to an ordered ferromagnetic Ising system, removes the degeneracy between the two magnetic states. Only the state with positive net magnetization remains stable. The two magnetic states coexist only at zero field. This is a direct consequence of the Gibbs phase rule.

It is known that in nonequilibrium systems, this is not necessarily the case 44,44. Namely when a symmetry breaking field is applied, the state unfavoured by the field may stay as a stable thermodynamic state. This is in violation of the Gibbs phase rule, which does not hold in nonequilibrium.

The 'bridge' model described in this section provides a clear example for this behaviour [40]. To demonstrate this point we introduce a symmetry breaking field by imposing boundary conditions which favour, say, the positively charged state, thus explicitly breaking the symmetry. More specifically, we consider an exclusion model where, instead of having boundary rates $\alpha, \beta$ for both types of particles, we take $\alpha, \beta_{+}$for the positive charges and $\alpha, \beta_{-}$ for the negative charges. The two exit rates are taken to be of the form $\beta_{ \pm}=\beta(1 \mp H)$, where $0<H<1$ is the symmetry breaking field, favouring the positively charged state. The analysis presented above in the limit $\beta \rightarrow 0$ may be repeated for non-vanishing field $H$ and the stability of the two phases may be analysed. Here again the dynamics is reduced to a diffusion process of the type (31) but with modifies rates (see Figure 7). Clearly the positively charged state is stable since it is favoured by the field. The question is whether 


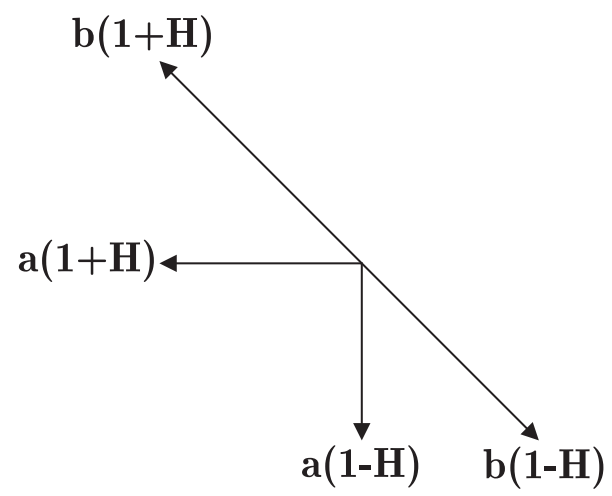

FIG. 7. The elementary moves of the biased diffusion process corresponding to the small $\beta$ limit when a symmetry breaking field $H$ is present.

the negatively charged state is stable when the field $H$ is non-vanishing. To examine this problem, we start with a negatively charged system $(N, 0)$ and consider a random walk defined by the diffusion process. It is easy to see that as long as $H<a /(1-a)=1 /(1+2 \alpha)$ the walk is biased in the negative $y$ direction, yielding a flipping time exponential in the system size. Thus the negatively charged state is stable even when it is unfavoured by the symmetry breaking field.

\section{Pase separation in one dimension}

A phenomenon closely related to spontaneous symmetry breaking is that of phase separation. In $1 d$ equilibrium systems with short range interactions phase separation does not take place, and therefore no liquid-gas like transition is expected. The density of particles in such a system is thus macroscopically homogeneous.

Recent studies have shown that driven systems may exhibit phase separation in $1 d$ even when the system is governed by local dynamics [39,45 47. Several models have been introduced to demonstrate this behaviour. In these models more than one type of particles is needed for phase separation to take place. In the following we consider in some detail one of these models, and analyse the mechanism leading to nonequilibrium phase separation [39].

The model is defined on a $1 d$ lattice of length $N$ with periodic boundary conditions. 
Each site is occupied by either an $A, B$, or $C$ particle. The evolution is governed by random sequential dynamics defined as follows: at each time step two neighbouring sites are chosen randomly and the particles of these sites are exchanged according to the following rates

$$
\begin{aligned}
& A B \underset{1}{\stackrel{q}{\rightleftarrows}} B A \\
& B C \underset{q}{\stackrel{q}{\rightleftarrows}} C B \\
& C A \stackrel{q}{\rightleftarrows} A C .
\end{aligned}
$$

The rates are cyclic in $A, B$ and $C$ and conserve the number of particles of each type $N_{A}, N_{B}$ and $N_{C}$, respectively.

For $q=1$ the particles undergo symmetric diffusion and the system is disordered. This is expected since this is an equilibrium steady state. However for $q \neq 1$ the particle exchange rates are biased. We will show that in this case the system evolves into a phase separated state in the thermodynamic limit.

To be specific we take $q<1$, although the analysis may trivially be extended for any $q \neq 1$. In this case the bias drives, say, an $A$ particle to move to the left inside a $B$ domain, and to the right inside a $C$ domain. Therefore, starting with an arbitrary initial configuration, the system reaches after a relatively short transient time a state of the type $\ldots A A B B C C A A A B \ldots$ in which $A, B$ and $C$ domains are located to the right of $C, A$ and $B$ domains, respectively. Due to the bias $q$, the domain walls $\ldots A B \ldots, \ldots B C \ldots$, and $\ldots C A \ldots$, are stable, and configurations of this type are long-lived. In fact, the domains in these configurations diffuse into each other and coarsen on a time scale of the order of $q^{-l}$, where $l$ is a typical domain size in the system. This leads to the growth of the typical domain size as $(\ln t) /|\ln q|$. Eventually the system phase separates into three domains of the different species of the form $A \ldots A B \ldots B C \ldots C$. A finite system does not stay in such a state indefinitely. For example, the $A$ domain breaks up into smaller domains in a time of order $q^{-\min \left\{N_{B}, N_{C}\right\}}$. In the thermodynamic limit, however, when the density of each type of particle is non vanishing, the time scale for the break up of extensive domains diverges and we expect the system to phase separate. Generically the system supports particle currents in 
the steady state. This can be seen by considering, say, the $A$ domain in the phase separated state. The rates at which an $A$ particle traverses a $B(C)$ domain to the right (left) is of the order of $q^{N_{B}}\left(q^{N_{C}}\right)$. The net current is then of the order of $q^{N_{B}}-q^{N_{C}}$, vanishing exponentially with $N$. This simple argument suggests that for the special case $N_{A}=N_{B}=N_{C}$ the current is zero for any system size.

The special case of equal densities $N_{A}=N_{B}=N_{C}$ provide very interesting insight into the mechanism leading to phase separation. We thus consider it in some detail. Examining the dynamics for these densities, one finds that it obeys detailed balance with respect to some distribution function. Thus in this case the model is in fact in thermal equilibrium. It turns out however that although the dynamics of the model is local the effective Hamiltonian corresponding to the steady state distribution has long range interactions, and may thus lead to phase separation. This particular mechanism is specific for equal densities. However the dynamical argument for phase separation given above is more general, and is valid for unequal densities as well.

In order to specify the distribution function for equal densities, we define a local occupation variable $\left\{X_{i}\right\}=\left\{A_{i}, B_{i}, C_{i}\right\}$, where $A_{i}, B_{i}$ and $C_{i}$ are equal to one if site $i$ is occupied by particle $A, B$ or $C$ respectively and zero otherwise. The probability of finding the system in a configuration $\left\{X_{i}\right\}$ is given by

$$
W_{N}\left(\left\{X_{i}\right\}\right)=Z_{N}^{-1} q^{\mathcal{H}\left(\left\{X_{i}\right\}\right)} .
$$

where $\mathcal{H}$ is the Hamiltonian

$$
\mathcal{H}\left(\left\{X_{i}\right\}\right)=\sum_{i=1}^{N} \sum_{k=1}^{N-1}\left(1-\frac{k}{N}\right)\left(C_{i} B_{i+k}+A_{i} C_{i+k}+B_{i} A_{i+k}\right)-(N / 3)^{2},
$$

and the partition sum is given by $Z_{N}=\sum q^{\mathcal{H}\left(\left\{X_{i}\right\}\right)}$. The value of the site index $(i+k)$ in (37) is taken modulo $N$. In this Hamiltonian the interaction between particles is long range, growing linearly with the distance between the particles.

In order to verify that the dynamics (35) obeys detailed balance with respect to the distribution function (36, 37) it is useful to note that the energy of a given configuration may be evaluated in an alternate way. Consider the fully phase separated state 


$$
A \ldots A B \ldots B C \ldots C
$$

The energy of this configuration is $E=0$, and, together with its translationally relates configurations, they constitute the $N$-fold degenerate ground state of the system. We now note that nearest neighbour (nn) exchanges $A B \rightarrow B A, B C \rightarrow C B$ and $C A \rightarrow A C$ cost one unit of energy each, while the reverse exchanges result in an energy gain of one unit. The energy of an arbitrary configuration may thus be evaluated by starting with the ground state and performing nn exchanges until the configuration is reached, keeping track of the energy changes at each step of the way. This procedure for obtaining the energy is self consistent only when the densities of the three species are equal. To examine self consistency of this procedure consider, for example, the ground state (38), and move the leftmost particle $A$ to the right by a series of nn exchanges until it reaches the right end of the system. Due to translational invariance, the resulting configuration should have the same energy as (38), namely $E=0$. On the other hand the energy of the resulting configuration is $E=N_{B}-N_{C}$ since any exchange with a $B$ particle yields a cost of one unit while an exchange with a $C$ particle yields a gain of one unit of energy. Therefore for self consistency the two densities $N_{B}$ and $N_{C}$ have to be equal, and similarly, they have to be equal to $N_{A}$.

The Hamiltonian (37) may be used to calculate steady state averages corresponding to the dynamics (35). We start by an outline of the calculation of the free energy. Consider a ground state of the system (38). The low lying excitations around this ground state are obtained by exchanging nn pairs of particles around each of the three domain walls. Let us first examine excitations which are localized around one of the walls, say, $A B$. An excitation can be formed by one or more $B$ particles moving into the $A$ domain (equivalently $A$ particles moving into the $B$ domain). A moving $B$ particle may be considered as a walker. The energy of the system increases linearly with the distance traveled by the walker inside the $A$ domain. An excitation of energy $m$ at the $A B$ boundary is formed by $j$ walkers passing a total distance of $m$. Hence, the total number of states of energy $m$ at the $A B$ boundary is equal to the number of ways $P(m)$ of partitioning an integer $m$ into a sum of (positive) 
integers. This and related functions have been extensively studied in the mathematical literature over many years. Although no explicit general formula for $P(m)$ is available, its asymptotic form for large $m$ is known 48

$$
P(m) \simeq \frac{1}{4 m \sqrt{3}} \exp \left(\pi(2 / 3)^{1 / 2} m^{1 / 2}\right)
$$

Also, a well known result attributed to Euler yields the generating function

$$
Y=\sum_{m=0}^{\infty} q^{m} P(m)=\frac{1}{(q)_{\infty}}
$$

where

$$
(q)_{\infty}=\lim _{n \rightarrow \infty}(1-q)\left(1-q^{2}\right) \ldots\left(1-q^{n}\right)
$$

This result may be extended to obtain the partition sum $Z_{N}$ of the full model. In the limit of large $N$ the three domain walls basically do not interact. It has been shown that excitations around the different domain boundaries contribute additively to the energy spectrum [39]. As a result in the thermodynamic limit the partition sum takes the form

$$
Z_{N}=N /\left[(q)_{\infty}\right]^{3}
$$

where the multiplicative factor $N$ results from the $N$-fold degeneracy of the ground state and the cubic power is related to the three independent excitation spectra associated with the three domain walls.

It is of interest to note that the partition sum is linear and not exponential in $N$, as is usually expected, meaning that the free energy is not extensive. This is a result of the longrange interaction in the Hamiltonian and the fact that the energy excitations are localized near the domain boundaries.

Whether or not a system has long-range order in the steady state can be found by studying the decay of two-point density correlation functions. For example the probability of finding an $A$ particle at site $i$ and a $B$ particle at site $j$ is,

$$
\left\langle A_{i} B_{j}\right\rangle=\frac{1}{Z_{N}} \sum_{\left\{X_{k}\right\}} A_{i} B_{j} q^{\mathcal{H}\left(\left\{X_{k}\right\}\right)},
$$


where the summation is over all configurations $\left\{X_{k}\right\}$ in which $N_{A}=N_{B}=N_{C}$. Due to symmetry many of the correlation functions will be the same, for example $\left\langle A_{i} A_{j}\right\rangle=$ $\left\langle B_{i} B_{j}\right\rangle=\left\langle C_{i} C_{j}\right\rangle$. A sufficient condition for the existence of phase separation is

$$
\lim _{r \rightarrow \infty} \lim _{N \rightarrow \infty}\left(\left\langle A_{1} A_{r}\right\rangle-\left\langle A_{1}\right\rangle\left\langle A_{r}\right\rangle\right)>0
$$

Since $\left\langle A_{i}\right\rangle=1 / 3$ we wish to show that $\lim _{r \rightarrow \infty} \lim _{N \rightarrow \infty}\left\langle A_{1} A_{r}\right\rangle>1 / 9$. In fact it can be shown [39] that for any given $r$ and for sufficiently large $N$,

$$
\left\langle A_{1} A_{r}\right\rangle=1 / 3-\mathcal{O}(r / N)
$$

This result not only demonstrates that there is phase separation, but also that each of the domains is pure. Namely the probability of finding a particle a large distance inside a domain of particles of another type is vanishingly small in the thermodynamic limit.

Numerical simulations of the model for the case of unequal densities, where such analysis cannot be carried out, strongly indicate that phase separation takes place as long as none of the three densities vanish. They also indicate that the coarsening process which accompanies phase separation is rather slow, with the characteristic length diverging like ln $t$ at long times.

\section{Summary}

In these lecture notes some collective phenomena which occur in one-dimensional driven systems have been reviewed. These systems have been extensively studied in recent years by introducing simple models and analysing their steady state properties. Some of these models have been demonstrated to exhibit a rich variety of phenomena which are unexpected in equilibrium one-dimensional systems.

Simple asymmetric exclusion processes in open systems were shown to exhibit both first order and continuous phase transitions. Other systems which have in the past been demonstrated to exhibit phase transitions in $1 d$ are directed percolation [49,50] and contact

processes [51]. These system, however, possess one or more absorbing states. Once the 
system evolves into one of these states the dynamics is such that the system is unable to exit. Under these conditions, the existence of a phase transition between a trapped and an untrapped states is rather natural. Usually, once the dynamics in these models is generalised to allow for an exit from the absorbing state no phase transition takes place. The phase transitions occurring in the asymmetric exclusion processes discussed in this paper are rather different, as the dynamics in these models does not possess absorbing states.

Mechanisms which lead to spontaneous symmetry breaking and phase separation in onedimensional nonequilibrium systems have been discussed. A common crucial feature of these models is that the dynamics conserves, at least to some degree, the order parameter. In the 'bridge' model, the densities of the two types of particles are conserved in the bulk although they are not conserved at the two ends of the system. In the $A B C$ model, on the other hand, the three densities are fully conserved. When non-conserving processes are introduced into these models spontaneous symmetry breaking and phase separation do not take place. It would be very interesting to consider the possibility of spontaneous symmetry breaking in one dimension when the dynamics does not conserve the order parameter. A related problem has been considered in the context or error correcting computation algorithems. An example of a one-dimensional array of coupled probabilistic cellular automata has been constructed and shown to yield breaking of ergodicity, as would a model with spontaneous symmetry breaking [52]. This approach suggests that indeed spontaneous symmetry breaking in $1 d$ may exist even when the dynamics is not conserving. However the example given is rather complicated and not well understood.

In spite of the progress made in recent years in the understanding of nonequilibrium collective phenomena, many basic questions remain open, even for the restricted and relatively simple class of systems which evolve into a steady state. For example a classification of continuous nonequilibrium transitions into universality classes, like the one which exists for equilibrium transitions, is not available. Also the dynamical process of the approach to steady state is far from being understood in many cases. The approach outlined in these notes, which involves constructing simple dynamical models and analysing the resulting col- 
lective behaviour may prove to be helpful in developing better understanding of some of these complex questions.

\section{Acknowledgments}

I thank Martin Evans and Yariv Kafri for many useful comments and for critical reading of the manuscript. 


\section{REFERENCES}

[1] Schmittmann B and Zia R K P, 1995, Phase Transitions and Critical Phenomena vol 17, editors C Domb and J Lebowitz (Academic Press, London).

[2] Krug J and Spohn H, 1991, in Solids Far From Equilibrium editor C Godrèche (Cambridge University Press, Cambridge).

[3] Halpin-Healy T and Zhang Y C, 1995, Physics Reports 254215.

[4] Krug J, 1997, Advances in Physics 46139.

[5] Proceedings of workshop on Traffic and Granular Flow 1995 editors D E Wolf, M Schreckenberg and A Bachem (World Scientific, Singapore)

[6] Nagel K and Schreckenberg M, 1992, J. Physique I 22221.

[7] Schreckenberg M, Schadschneider A, Nagel K and Ito N, 1995, Phys. Rev. E 512339.

[8] Alberts B, Bray D, Raff M, Roberts K and Watson J D, 1994, Molecular Biology of the Cell, 3rd Edition (Garland Publishing, New York).

[9] Duke T A J, 1990 J. Chem. Phys. 93 9052; Duke T A J and Viovy J L, 1992, Phys. Rev. Lett. 68542.

[10] Geller S, 1977, Solid Electrolytes, Topics in Applied Physics, Vol. 21 (Springer, Heidelberg).

[11] Salamon M B, 1979, Physics of Superionic Conductors, Topics in Current Physics, Vol. 15 (Springer, Heidelberg).

[12] Wilson K G and Kogut J, 1974, Physics Reports 12C 75.

[13] Fisher M E, 1998, Rev. Mod. Phys. 70653.

[14] Landau L D and Lifshitz E M, 1980, Statistical Physics I (Pergamon Press, New York).

[15] Mermin N D and Wagner H, 1966, Phys. Rev. Lett. 171133. 
[16] de Gennes P G and Prost J, 1993, The Physics of Liquid Crystals 2nd edition (Clarendon Press, Oxford).

[17] Wheeler J, 1974, J. Chem. Phys. 614474.

[18] Harris A B, 1974, J. Phys. C 71671.

[19] Imry Y and Ma S K, 1975, Phys. Rev. Lett. 351399.

[20] Katz S, Lebowitz J L and Spohn H, 1983, Phys. Rev. B28 1655; 1984, J. Stat. Phys. 34497.

[21] MacDonald J T, Gibbs J H and Pipkin A C, 1968, Biopolymers 61.

[22] MacDonald J T and Gibbs J H, 1969, Biopolymers 7707.

[23] Krug J, 1991, Phys. Rev. Lett. 671882.

[24] Derrida B, Domany E and Mukamel D, 1992, J. Stat. Phys. 69667.

[25] Derrida B, Evans M R, Hakim V and Pasquier V, 1993 J Phys A: Math. Gen. 26 1493;

1993, in Cellular Automata and Cooperative Systems editors N Boccara et al, (Kluwer Academic Publishers) 121.

[26] Schütz G M and Domany E, 1993, J. Stat. Phys. 72277.

[27] Kolomeisky A B, Schütz G M, Kolomeisky E B and Straley J P, 1998, J Phys A: Math. Gen. 316911.

[28] Hinrichsen H, 1996, J Phys A: Math. Gen. 293659.

[29] Evans M R, Rajewsky N and Speer E R, 1999, J. Stat. Phys. 9545.

[30] de Gier J and Nienhuis B, 1999, Phys. Rev. E 594899

[31] Sandow S, 1994, Phys. Rev. E 502660.

[32] Sasamoto T, 1999, J Phys A: Math. Gen. 327109. 
[33] Blythe R A, Evans M R, Colaiori F and Essler F H L, 1999, cond-mat/9910242.

[34] Derrida B, Janowsky S A, Lebowitz L and Speer E R, 1993, Europhys. Lett. 22 651; Mallick K, 1996, J Phys A: Math. Gen. 295375.

[35] Evans M R, 1996, Europhys. Lett. 3613.

[36] Arndt P F, Heinzel T and Rittenberg V, 1998, J Phys A: Math. Gen. 31833.

[37] Evans M R, Foster D P, Godrèche C, and Mukamel D, 1995, Phys Rev Lett 74 208; 1995, J. Stat. Phys. 8069.

[38] Derrida B, Evans M R and Mallick K, 1995, J. Stat. Phys. 79833.

[39] Evans M R, Kafri Y, Koduvely H M and Mukamel D, 1998, Phys. Rev. Lett. 80 425; 1998, Phys. Rev. E 582764.

[40] Godrèche C, Luck J-M, Evans M R, Mukamel D, Sandow S and Speer E R, 1995, J Phys A: Math. Gen. 286039.

[41] Arndt P F, Heinzel T and Rittenberg V, 1998, J. Stat. Phys. 90783.

[42] Arndt P F and Heinzel T, 1998, J. Stat. Phys. 92837.

[43] Toom A L, 1980, in Multicomponent Random Systems editor R L Dobrushin, in Advanced in Probability, Vol. 6 (Dekker, New York) 549.

[44] Bennett C H and Grinstein G, 1985, Phys. Rev. Lett. 55 657.ABC,

[45] Lahiri R and Ramaswamy S, 1997, Phys. Rev. Lett. 791150.

[46] Lahiri R, Barma M and Ramaswamy S, 2000, Phys. Rev. E61 1648.

[47] Arndt P F, Heinzel T and Rittenberg V, 1998, J Phys A: Math. Gen. 31 L45; 1999, J. Stat. Phys. 971

[48] Andrews G E, 1976, The Theory of Partitions, Encyclopedia of Mathematics and its 
Applications (Addison Wesley, MA), 2 1-4.

[49] Domany E and Kinzel W, 1984 Phys. Rev. Lett. 53311.

[50] Kinzel W, 1985, Z. Phys. B 58229.

[51] Durrett R and Liggett T M, 1981, Ann. Probab. 18 186; Jensen I and Dickman R, 1993 J. Phys. A: Math. Gen. 13 L423.

[52] Gacs P, 1986, J. Comput. Sys. Sci. 3215. 